\title{
MODIFICAÇÕES EM JOGOS DIGITAIS E SEU USO POTENCIAL COMO TECNOLOGIA EDUCACIONAL PARA O ENSINO DE ENGENHARIA
}

\author{
Luis Otoni Meireles Ribeiro, PPGIE-UFRGS, CEFET-RS, luis.otoni@terra.com.br \\ Maria Isabel Timm, PPGIE-CESUP-UFRGS, betatimm@ufrgs.br \\ Milton Antonio Zaro, PPGIE-UFRGS, zaro@ufrgs.br
}

Resumo: Este artigo apresenta as modificações de jogos digitais como uma alternativa viável e eficaz de tecnologia educacional e revisa algumas pesquisas sobre o uso de jogos comerciais em atividades de educação e treinamento. Os mod-games ao possibilitarem a construção de cenários educacionais e laboratórios virtuais, e seu uso de forma interativa, imersiva e colaborativa, elevam a experimentação pelos alunos a um novo nível ao permitirem o desenvolvimento de habilidades cognitivas contextualizadas com as exigências atuais da educação e, em especial, do ensino de engenharia. Ao reduzir o ciclo de desenvolvimento de jogos as modificações de games tornam-se mais acessíveis aos professores, permitindo a pesquisa educacional na área.

Palavras-Chave: jogos educacionais, simulação, laboratórios virtuais, ensino de ciência e tecnologia, ensino de engenharia

Abstract: This paper presents the use of mod-games (digital games modifications) as an effective educational technology, and summarizes main researches about the use of commercial games for training and educational goals. The mod-games allow the construction of educational environments and virtual laboratories. Its use, in interactive, immersive and collaborative way, increases the level of the student's development of cognitive skills, attending contemporary education's requirement, in particular for strongly demanding areas, like engineering. By reducing development cycle of the educational games, these modifications make them more accessible and contribute to improve educational research.

Key-Words: educational games, simulation, virtual laboratory, science and technology education, engineering education

\section{Introdução}

As pesquisas na área de tecnologias educacionais atravessam um período em que a diversidade tecnológica se destaca. Em especial, o uso da informática na educação descortina um imenso campo de possibilidades, sendo possível destacar um campo específico neste segmento, o dos jogos digitais. $\mathrm{O}$ uso de games na educação tem sido objeto de diversos estudos. Sugere-se, a este respeito, que o ensino de ciência e tecnologia, e especificamente, o ensino de engenharia, no Brasil, pode se beneficiar com a pesquisa sobre o potencial desse recurso como apoio ao processo educacional. $\mathrm{O}$ objetivo desse artigo é revisar o estado da arte na produção de cenários e laboratórios educacionais a partir de modificações de jogos (games) digitais comerciais e apresentar estudos acerca de sua utilização como tecnologias educacionais viáveis, pela sua natureza pedagógica cognitiva, e pela otimização do ciclo de produção que oferecem aos produtores e professores.

$\mathrm{O}$ setor de jogos digitais vive uma fase de crescimento recorde, graças ao enorme volume financeiro que recebe atualmente, com uma estimativa de fluxo para 2007 de US\$ 21 bilhões (DTI, 2006), superando a indústria cinematográfica. Nesse mercado bilionário as empresas produtoras de games têm conseguido contratar profissionais qualificados e incorporar tecnologias de uma forma muito intensa 
(Azevedo, 2005). Poucos segmentos da área de desenvolvimento de software conseguiram absorver tão rapidamente os avanços nas pesquisas de informática como os desenvolvedores de games (Perucia, 2005). Pesquisas relacionadas a Inteligência Artificial (IA), Redes Neurais, Sistemas Multiagentes e Física são temas presentes nos congressos de jogos. Os desenvolvedores, para minimizar os custos de criação de seus produtos, implementam os chamados game engine, ou motores de jogos, um "conjunto de componentes de software integrados e reutilizáveis projetados para facilitar o desenvolvimento de jogos de computadores" (Nakamura 2003).

Os motores de jogos são modularizados para tratar de funções específicas no ciclo de desenvolvimento dos games. Existem os motores gráficos responsáveis por toda a parte de gráfica do jogo, com seus cenários e texturas, assim como motores de IA e motores de física (Hecker, 2000), que facilitam o desenvolvimento das ações dos jogos, facilitando a programação e a simulação de fenômenos da realidade. Essa modularização permite a separação no desenvolvimento do jogo entre a parte artística, o roteiro do jogo com seus scripts, que compõem a parte lógica, e a parte de programação dos motores em si. As grandes empresas de desenvolvimento optam por desenvolver seus próprios motores, mas alguns desenvolvedores optam por licenciar engines e trabalham na implementação de seus argumentos e histórias. Um bom exemplo desse funcionamento é a franquia desenvolvida pelo diretor norte-americano George Lucas, com a empresa Lucas Arts (http://www.lucasarts.com/) que traduziu em diversos títulos de games a série cinematográfica Star Wars.

No que se refere a physics game engine, ou seja, ao motor de desenvolvimento da física de jogos, destaca-se a HAVOC (http://www.havok.com/products/physics.php). Sua engine está disponível para a maioria das plataformas de games. A engine de física realística Havoc2 permite a interação dos jogadores com objetos físicos, como por exemplo seixos, os quais, se atirados na água, comportam-se, como esperado, tal qual objetos pesados, obedecendo às leis de massa, fricção, gravidade, e flutuação. Um exemplo de integração da engine de física Havoc2 com um jogo, é o Half-Life 2 da empresa VALVE, que incorpora a Havoc 2 com sua própria engine de renderização Source. O jogo propicia um elevado grau de liberdade na exploração interativa dos objetos presentes no ambiente.

Half-Life 2 não é o primeiro jogo a usar a engine de física realística do Havoc 2, mas certamente acrescentou valor ao integrar o efeito realístico dos fenômenos aos atrativos da interatividade. Algumas cenas podem ser experimentadas de várias formas, dependendo da criatividade dos jogadores na sua relação com o ambiente e com os objetos disponíveis. Existem atualmente cerca de 120 títulos que licenciaram o Havok para plataformas Xbox, Playstation, PC e Gamecube. Baseado nesta gama de possibilidades, foi adotado o Half-life 2 como plataforma de game para exploração de experimentos educacionais, em projeto de tese atualmente em fase de implantação junto ao Programa de Pós-Graduação em Informática na Educação da UFRGS ${ }^{1}$. Ao longo do artigo, serão mostradas modificações baseadas nesse jogo com fins educacionais, que exploram as potencialidades e as limitações de seu uso.

\section{Jogos Digitais como Ferramentas Pedagógicas}

Os jogos digitais, ao permitirem a simulação em ambientes virtuais, proporcionam momentos ricos de exploração e controle dos elementos. Neles, os jogadores - crianças, jovens ou adultos - podem explorar e encontrar, através de sua ação, o significado dos elementos conceituais, a visualização de situações reais e os resultados possíveis do acionamento de fenômenos da realidade. Ao combinar diversão

\footnotetext{
${ }^{1}$ O referido projeto de tese está sendo desenvolvido pelo primeiro autor do presente artigo, sob orientação do prof. Dr. Milton Antonio Zaro.

$2 \longrightarrow$ V. 4 No 1, Julho, 2006
} 
e ambiente virtual, transformam-se numa poderosa ferramenta narrativa, ou seja, permitem criar histórias, nas quais os jogadores são envolvidos, potencializando a capacidade de ensino-aprendizado. Murray (1997) atribui a esta nova narrativa quatro propriedades importantes: procedurais (porque podem ser programados para executar tarefas e inferências, com base em regras e padrões), participativos (porque permitem que os usuários realizem atividades, desafios, etc.), espaciais (porque os usuários atuam neles de dentro da própria cena) e enciclopédicos (porque podem estar integrados à biblioteca global da Internet, além de bases específicas de conhecimento). Esses ambientes encantam por incorporar às narrativas tradicionais três novas possibilidades de envolvimento do jogador-navegador: imersão, agenciamento e transformação /simulação.

A imersão em games depende da percepção e da ação do sujeito. Pesquisas cognitivas sugerem que a percepção humana e a ação estão profundamente interconectadas (Gee, 2004). Ao jogar um game, quando uma pessoa dirige um carro ou abre uma porta no porão do castelo, ela sente como se o seu corpo e mente estivessem inseridos em um novo espaço. Por isso, o jogador sente-se livre e interessado, em explorar livremente o ambiente, interagindo com os elementos. Ao se auto-representar internamente em um cenário virtual, o jogador sente prazer, através do que vem sendo descrito como uma sensação, ou percepção, de imersão, ou seja, ele está envolto pela ação, pelos elementos do cenário e pelos desafios apresentados ao personagem que está representando. Essa sensação se relaciona com a sensação de agenciamento, pois o jogador quer agir, atuar nesse cenário, deixar a posição de observador passivo e ser um agente protagonista que vê o resultado de suas ações e escolhas.

No caso da característica de agenciamento, que pode contribuir para a eficiência e a verossimilhança dos games, a ação do jogador produz uma conseqüência, cujos resultados interferem na narrativa, o que permite avaliar suas próprias escolhas e tomadas de decisão. Esta característica, associada à de transformação e simulação, permite ao jogador sentir-se poderoso e responsável. Afinal, onde mais poderia decidir, por exemplo, sobre o futuro da humanidade, vivenciar atos heróicos, resgatando reféns, mudando o curso da história. Caso as escolhas resultem em derrota, basta recomeçar o jogo e passar pela mesma experiência mas fazendo escolhas diferentes, muitas vezes pelo simples prazer de fazer diferente ou responder aquela curiosidade "e se eu fizesse assim....".

A geração atual de crianças e adolescentes tem gasto mais tempo semanal jogando games do que estudando e fazendo seus deveres escolares (Hostetter, 2006). Essas pesquisas mostram que esse uso constante tem modificado as habilidades cognitivas dessa geração: ao consultar um material, primeiro eles examinam os gráficos e depois lêem os textos para adicionar informações. Sentem-se à vontade com informações hipertextuais, com a exigência de habilidades visuais-espaciais, constroem mapas mentais e usam o computador como uma ferramenta mental.

Segundo Mayo (2005) o uso de games para treinar, aprender e executar atividades reais em ambientes realísticos melhora a performance dos aprendizes, tornam-se melhores através da aprendizagem baseada em games. Possibilitam experiências de aprendizagem produzidas individualmente de acordo com seu estilo de aprendizagem e desempenho. A pesquisa é bem completa e apresenta diversas pesquisas sobre a influência dos jogos digitais na aprendizagem, inclusive com estudos de neurociência. Seu estudo compara as teorias de aprendizagem com características dos jogos:

“- Aprendizagem experimental (você faz, você aprende): participação ativa com decisões que tem conseqüências. Típico de jogos imersivos; 
- Aprendizagem baseada no questionamento e feedback (o que acontece quando eu faço isto?): exploração em jogos;

- Autenticidade (quanto mais a situação de aprendizagem for realista, mais facilmente os aprendizes transferem a informação para a vida real): mundos virtuais;

- Eficácia própria (se você acredita que você pode fazer você aumenta suas chances de sucesso): recompensas e níveis nos games;

- Estabelecer metas (você efetua um progresso maior se trabalhar com metas bem definidas): objetivos do jogo;

- Cooperação (aprendizagem em time) - estudos mostram que a aprendizagem cooperativa apresenta resultados 50\% superiores sobre a aprendizagem individual ou competitiva: jogos massivamente multiusuário - MMOGs.” (Mayo, 2005)

$\mathrm{O}$ uso de jogos digitais como ferramenta para potencializar o ensino tem sido demonstrada em vários estudos. A ênfase do uso dos games aparece com focos distintos. Um grande número de trabalhos destaca o desenvolvimento de jogos digitais como facilitador do ensino de ciência da computação. A investigação de Silva e Silva (2005) relata experiências tais como o desenvolvimento de jogos de computadores baseados em interface Web, que facilita o ensino de conceitos fundamentais de computadores; um curso de projeto e implementação de jogos digitais, que provê um ambiente ideal para os estudantes integrarem uma gama de conhecimentos e habilidades de ciências da computação; e na pós-graduação na área, um curso de Inteligência Artificial (IA), no qual são construídos jogos mais realísticos e interessantes e que permitem excelentes cenários nos quais se podem testar e aplicar as técnicas de IA aprendidas. Entretanto, essas abordagens tratam do desenvolvimento de jogos digitais, com ênfase na programação, como uma solução para potencializar o cenário educacional, motivando e engajando o aluno no processo educacional. Os jogos digitais seriam assim desenvolvidos usando linguagens de programação ou ferramentas de autoria, normalmente freeware que possibilitem o desenvolvimento dos jogos sem o pagamento de royalts.

Outra área relacionada aos jogos digitais que vem merecendo atenção da comunidade acadêmica é o uso de games comerciais na sala de aula, explorando ferramentas pedagógicas que se aplicam ou apóiam o desenvolvimento de disciplinas convencionais, como matemática ou geografia entre outras. Uma pesquisa sobre o potencial educativo do videogame foi conduzida utilizando o simulador de cidades SimCity4, envolvendo professores de Português, Matemática e Geografia (Ilha e Cruz, 2005). A pesquisa explora e argumenta sobre a necessidade dos professores adequarem seus conteúdos e estratégias didáticas de suas disciplinas para o ambiente multimídia, onde ocorrem mudanças nos processos de gerenciamento e regulação das situações de aprendizagem em função do jogo digital. A mudança principal é a troca de um processo tradicional de ensino para um que alie diversão e aprendizagem, facilitando uma aprendizagem por descobertas. Os games produzem mudanças nas habilidades cognitivas dos aprendizes, em especial a capacidade de processar uma quantidade maior de informação ao mesmo tempo e aprender através da experimentação e ações rápidas. De acordo com Hostetter (2005), citado no estudo, os games também desenvolvem o raciocínio dedutivo, estratégias de memorização e coordenação visual e motora. $\mathrm{O}$ estudo favoreceu a vivência de professores e alunos de uma experimentação interdisciplinar, outro efeito foi o da abertura do grupo para novas alternativas didáticas.

A compreensão de que as ferramentas para desenvolvimento de jogos trazem em si outros potenciais aparece no trabalho de Boron (2006) que argumenta que a indústria de games oferece uma poderosa ferramenta de expressão, com a qual tanto arquitetos quanto designers de jogos podem experimentar seus projetos através de um ambiente 
virtual, imersivo e interativo em tempo-real. A pesquisa busca diluir as distinções entre as ferramentas que arquitetos e designers utilizam.

Um outro estudo a ser destacado é o dos professores do SENAC-SP (Tavares e Neves, 2005) que tem trabalhado os fundamentos de game design através de modificações de jogos digitais comercias, no caso o Half-Life, a utilização dessa estratégia de modificação em jogos conhecidos mostram o que são e como os principais conceitos em design de jogos podem ser aplicados, permitindo aos alunos desenvolver e aplicar conceitos a um custo baixo e com resultados rápidos e atraentes.

Ao reunir ambientes realísticos, interação e imersividade, os mundos virtuais dos jogos enfraquecem a distinção entre experiência física e virtual. Esses espaços de jogos ao serem apresentados perceptivamente em primeira pessoa recriam a experiência construtiva do ambiente. Além disso, ao aliar-se a interação em tempo real entre indivíduos através de um jogo baseado em internet, os ambientes de jogos mostram seu potencial numa função considerada chave e associada à arquitetura, que é a criação de lugares significativos para encenar e suportar trocas sociais. Segundo o autor dessa pesquisa "quando esses lugares virtuais são reconectados ao real, através de vídeos digitais em tempo real, os ambientes virtuais podem funcionar como pontos de acesso, ou portais, de volta à realidade" (Boron, 2006, p.1). O trabalho citado aponta para os objetivos da pesquisa desenvolvida pelos autores deste artigo, na qual os ambientes projetados para funcionar como jogos educacionais recriam situações problemas para o estudante de engenharia, potencializando a interação num ambiente de aprendizagem virtual.

\section{Modificações de Jogos Digitais - um novo cenário}

As empresas de games, interessadas em ampliar o número de usuários de seus produtos, têm disponibilizado ferramentas para a construção de modificações para jogos (Mod-Games). Desta forma, juntamente com a engine disponibilizada, são reunidas outras ferramentas auxiliares que se destinam à criação de cenários ou personagens, com isso os próprios usuários ou outras empresas podem desenvolver modificações (mods) sobre o jogo original.

Como o mod é um game que se utiliza do núcleo tecnológico de outro jogo digital, diversas empresas oferecem gratuitamente, via Internet, o suporte, tutoriais e os arquivos necessários para a construção de mods. No caso do Half-Life 2 (da empresa Valve), a empresa disponibiliza o Source Engine para a criação de mods, o qual inclui um kit (Source SDK) com: ferramenta de expressão facial - Face Poser, editor de mapas estilo WYSIWYG - Valve Hammer Editor, pré-visualizador completo de modelos - Half-Life Model Viewer, e outros recursos. A Valve distribui este kit através de sua ferramenta de autenticação e distribuição - Steam, bastando para isso o usuário dispor de um game original e registrar-se no ambiente (o download do kit é gratuito).

A facilidade em desenvolver modificações de jogos deve ser analisada em maior profundidade pela comunidade acadêmica no Brasil. As experiências atuais com modgames ainda se concentram no ensino de computação ou design de jogos. Entretanto o potencial de desenvolvimento não se limita a esses cenários, em outros países a construção de cenários e laboratórios virtuais através de modificações de jogos está mais avançada. O potencial para atividades de simulação e treinamento são inúmeras, tendo despertado o interesse de educadores (Aldrich, 2004).

"A modificação de jogos desponta como uma solução atraente, já que a implementação trabalha normalmente com jogos que já fazem parte do repertório dos educandos, e que já são efetivamente jogados. Com isso o educador não necessita preocupar-se com a resistência comum aos jogos 
educativos, contando também com a experiência de utilização do jogo que pode ser conhecida." (Neves, 2005)

Ao unir-se a possibilidade de simulação em cenários 3D com tecnologias familiares a geração atual de estudantes, é iniciada a convergência para uma nova tecnologia educacional, a dos jogos de simulação. Nesses jogos, o objetivo não é o de recriar sistemas de realidade virtual que procurem reproduzir os ambientes físicos com o maior realismo possível, este é o papel dos simuladores profissionais. Para Tori (2005)

"a rigor, para ser chamado de simulador, um determinado sistema deveria atender aos requisitos máximos de precisão quanto aos fenômenos que se tenha a intenção de levar ao virtual. Mas se considerarmos que os requisitos de simulação para jogos são diferentes, com menos exigência de precisão, podemos aceitar a categoria jogos de simulação".

A preocupação presente nesse trabalho é explorar as modificações de jogos como uma alternativa de baixo custo para a criação de cenários educativos e laboratórios em realidade virtual. Utiliza-se assim dos jogos de simulação para uma representação funcional da realidade, ainda que de forma simplificada, mas que permite "uma mistura entre as características de um jogo - competição, cooperação, regras etc - com aquelas de um simulador, que é a incorporação de características críticas da realidade" (Galvão et al, 2000).

A pesquisa em curso utilizará a ferramenta para criação de mapas - Valve Hammer Editor, que permite a criação de modificações do jogo Half-Life 2, na construção de um cenário urbano virtual, com diversas edificações, permitindo o estudo de patologias prediais. A Figura 1 retrata algumas das telas do cenário piloto. Cabe salientar que a arma que aparece na foto é um recurso de edição do próprio jogo, que será modificada na versão final por uma planilha de inspeção, ou outro elemento relacionado ao tema em pauta.

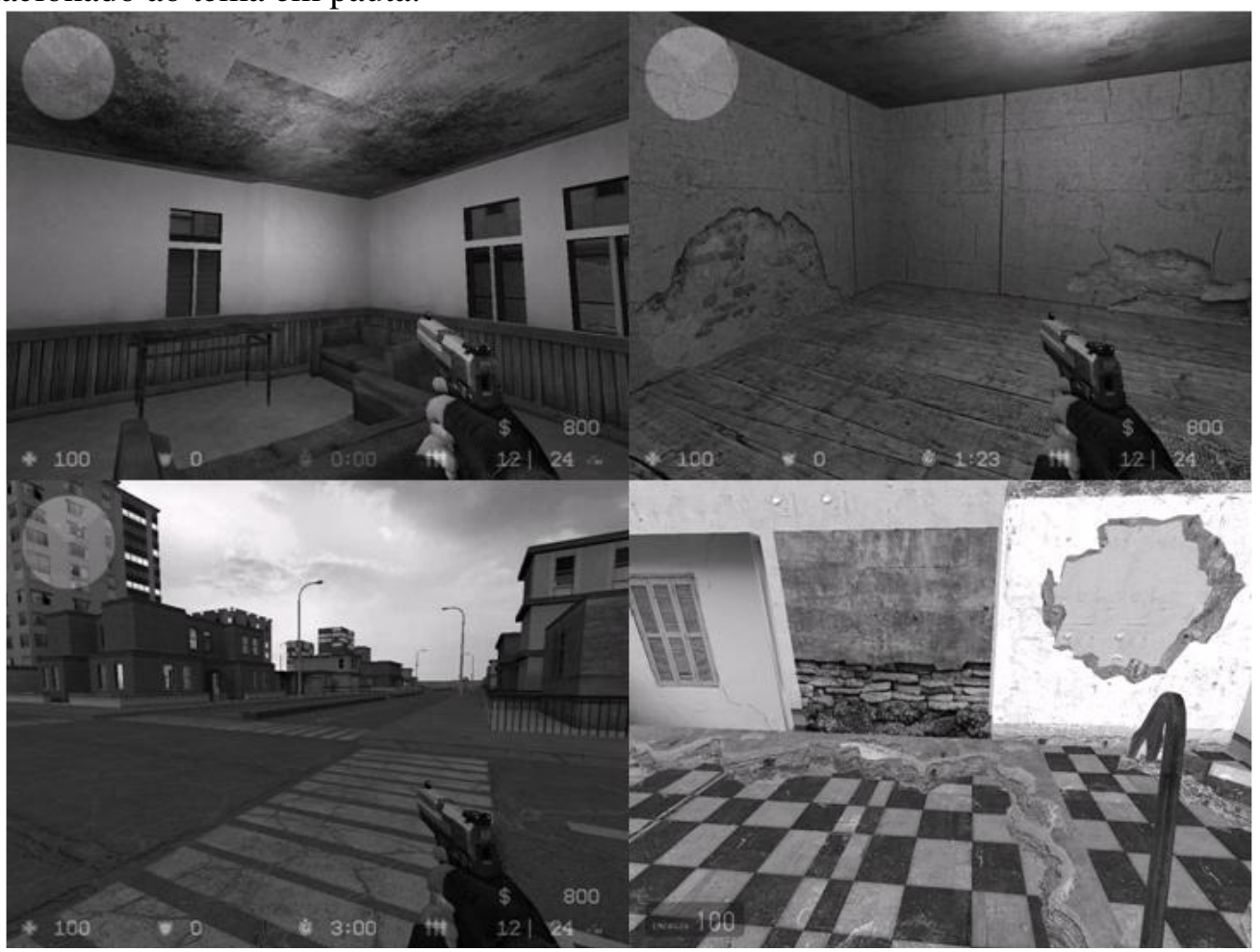

Figura 1 - Telas capturadas do cenário piloto com patologias prediais

$\mathrm{O}$ uso de ambientes 3D para o estudo de patologias foi sugerido em outra pesquisa (Hounsell et al, 2005). Entretanto o foco daquela pesquisa é na integração de textos e ambientes 3D interativos com propósitos educacionais, sem a utilização de 
mods. Já a pesquisa proposta busca inovar o ensino de patologias, através da oferta de uma ferramenta educacional que possa levar o aluno a desenvolver raciocínio diagnóstico e tomadas de decisões, com base em observação visual de casos reais simulados em ambiente 3D imersivo e desafios apresentados na linguagem de game usando motores gráficos de jogos comerciais.

É importante destacar que o game Half-Life 2 não se enquadra na categoria de jogos de simulação, ele é classificado como jogo de tiro em primeira pessoa (First Person Shooter - FPS), um jogo de ação. Entretanto, pela facilidade e recursos disponibilizados no source engine para criação de MODs, ele será o objeto de estudo inicial. O uso da game engine do Half-Life com fins educacionais não é inédito. Esta ferramenta já foi utilizada para implementar um laboratório virtual de simulação de acidentes (Bell e Floger, 2003), no qual os pesquisadores exploraram uma variedade de diferentes plataformas de desenvolvimento, para determinar quais seriam mais práticas e efetivas para simulação educacional em primeira pessoa. O estudo também destacou que os MODs viabilizam produção de realidade virtual a baixo custo, embora não atingindo um mesmo nível de realismo e credibilidade. Entretanto, seu uso tem como vantagem o acesso de um grande número de usuários, e, com os recentes avanços dos PCs e das placas gráficas, tornou-se possível aos estudantes acessar estes ambientes nas suas próprias universidades ou em casa, via Internet.

Um outro pesquisador tem se destacado no uso educacional de mods: Tim Holt (2005), como pesquisador assistente da Oregon State University desenvolve com pesquisadores florestais um visualizador de ambientes florestais, o GNN Visualization (GNNVis); usando a Valve Source Engine, em outro projeto para a Texas A\&M University Corpus Christi's desenvolve para o Office of Naval Research um ambiente para simulação e treinamento de atendimento de saúde de emergência, o projeto Pulse!, com a adoção não só do ambiente mas da própria linguagem de games. Ambos os projetos baseiam-se na modificação do jogo Half-Life 2 e contam com verbas federais norte-americanas para o seu desenvolvimento. Os dois estudos exploram a capacidade da ferramenta de permitir o desenvolvimento rápido de novos ambientes, incluindo apoio para ações disparadas pelos movimentos dos jogadores e interações com objetos que obedecem às leis da física. Também viabilizam a programação de características humanas para interagir com os humanos nos laboratórios de simulação. A Source Engine SDK também oferece um grande número de componentes úteis, incluindo objetos e mobília, como poltronas, cadeiras e computadores.

Outro recurso que não deve ser desconsiderado é a possibilidade de gravação das imagens do cenário virtual com câmeras de vídeo virtuais. Estas imagens poderiam servir para posterior análise pedagógica, pelo professor e alunos, do desenvolvimento das experiências no laboratório de realidade virtual. Aliando a possibilidade da Source Engine em criar MODs para até 32 usuários simultâneos, tem-se um recurso poderoso para proporcionar uma imersão de uma turma de alunos, como no cotidiano de sua vida escolar. Os experimentos podem ser executados de forma colaborativa, com os alunos discutindo entre si seus cálculos e previsões, observando o resultado de suas ações, e isso abre novas discussões sobre os impactos pedagógicos desses cenários virtuais.

$\mathrm{Na}$ pesquisa em desenvolvimento para o ensino de engenharia os primeiros cenários virtuais e laboratórios criados com mods demonstram que as texturas mapeadas para superfícies das construções apresentam o realismo necessário. Foram mapeadas imagens reais de patologias existentes em construções civis que foram aplicadas às construções virtuais. O processo de identificar patologias no ensino de engenharia envolve etapas complexas. Durante uma vistoria, por exemplo, o técnico procura por indícios e sintomas da ocorrência de algum fenômeno que possa prejudicar o bom 
desempenho das pessoas, das máquinas e equipamentos ou dos componentes da edificação. As dificuldades nessa capacitação envolvem:

- Sazonalidade ou temporalidade das obras com patologias;

- Desenvolvimento de raciocínios sobre casos práticos;

- Segurança e dificuldades de visualização;

- Diversidade de tipos de patologias.

O desenvolvimento de um cenário virtual através de uma modificação de jogos traz os seguintes benefícios iniciais:

- Recria cenários de difícil acesso, temporários e perigosos;

- Possibilita criar num espaço virtual localizado uma diversidade de situaçõesproblemas;

- Permite simular o processo de inspeção, vistoria, perícia e outros relacionados;

- Otimiza a visita virtual aos locais, a qualquer hora, circunstância, a distância (EAD) e de forma segura, dispensando o acompanhamento do professor;

- Permite a construção de situações problema contextualizadas.

A pesquisa irá explorar se é possível simular nos alunos, através da navegação no ambiente virtual de imersão, percepções e raciocínios equivalentes aos da percepção/análise visual e verificação in loco do cenário da patologia. Outra questão é testar se a interação com os personagens virtuais e a proposição de tarefas/desafios contribui para o engajamento dos estudantes na atividade pedagógica, ou seja, verificar se o roteiro, o argumento e a narrativa do jogo potencializam a ação pedagógica da tecnologia educacional adotada. As primeiras evidências apontam que os cenários 3D preservam os cases de construções reais e reúnem uma diversidade de patologias num cenário controlado e de fácil acesso, caracterizando, por isso, uma tecnologia educacional importante para o ensino de engenharia e, em especial, para o estudo de patologias de construções. Assim como, as ferramentas para criação de modificações de jogos digitais facilitam o ciclo de desenvolvimento dos ambientes educacionais 3D imersivos para o estudo das patologias de construção. Os resultados apresentados são iniciais e serão validados através de metodologia adequada.

\section{Conclusões e Perspectivas}

O recurso de Multiplayer disponível nos MODs abre espaço para diversas experimentações pedagógicas. Reforçando a tese de criarem-se ambientes de realidade virtual a um custo baixo, tem-se na criação de serious mods uma possibilidade que não deve ser dispensada. Se a simulação em primeira pessoa já traz em si mesma um grande atrativo para o estudante, que atua de forma ativa no ambiente, facilitando a construção de aprendizagens significativas; deve-se pensar então nas vantagens de permitir que os estudantes entrem nesse ambiente em grupos (Aldrich, 2005).

A aprendizagem cooperativa poderia ocorrer de forma espontânea com vários alunos realizando simultaneamente os experimentos (Gee, 2003). Deve-se lembrar as vantagens do modo multiplayer nos MODs, a primeira consiste na possibilidade de criação de avatares 3D personalizados, ou seja, pode-se, após escanear previamente a imagem do rosto dos alunos, atribuir estas imagens ao rosto de personagens individualizados dentro do ambiente. Atualmente os jogadores gastam um bom tempo personalizando as skins (imagens) de seus personagens virtuais, para que sejam reconhecidos e tenham o seu status diferenciado no game. Pedagogicamente o recurso de criarmos uma identidade visual para a presença virtual no ambiente reforçaria o sentimento de imersão na realidade virtual (Lazzaro, 2005). Aliando-se a isto o recurso de comunicação por voz e escrita, dispõe-se de canais efetivos pra recriar a atmosfera coletiva e participativa com a maior riqueza possível. 
O uso de motores (engines) de jogos comerciais pode ser questionado em função de ser uma engine proprietária, porém o escopo deste artigo é destacar exatamente a facilidade da comunidade educacional em se apropriar da tecnologia de criação de modificações de jogos e poder explorar os impactos de seu uso na educação. Vale a pena lembrar que a comunidade virtual existente produz e disponibiliza uma grande quantidade de informações, conteúdos e materiais para a criação de mods. A variedade de tutoriais e arquivos com exemplos e componentes é imensa, o que facilita a curva de aprendizado para os desenvolvedores informais, que são os educadores e alunos, assim como aceleram o ciclo de desenvolvimento pela utilização de componentes livremente disponibilizados.

Futuramente, como continuidade da pesquisa, será testado o envolvimento dos próprios alunos no processo de criação das modificações de jogos. Há evidências (Bell, 2004) do engajamento dos estudantes e de benefícios educacionais decorrentes dessa participação ativa no desenvolvimento de experiências imersivas. Os alunos podem envolver-se não só na pesquisa dos problemas de conteúdos a serem enfocados, como também na criação do storyboard, personagens, diálogos, geração de imagens e texturas de patologias reais, exploração e identificação de cases existentes a serem convertidos para o cenário digital. Assim, os estudantes assumiriam um papel de co-autores, superando o papel de simples atores para criar variantes dos jogos, o que ensejaria uma aprendizagem mais significativa uma vez que se pressupõe a autoria como umas características essenciais a uma aprendizagem autônoma e significativa.

A questão central nesse momento é testar limites e possibilidades dos argumentos para a área educacional, em especial o ensino de engenharia. Com certeza, após a comprovação das vantagens do seu uso, as aplicações validadas poderão ser portadas para engines gratuitas e feito um esforço de programação para a implementação dos recursos necessários, disponíveis nas engines proprietárias e comerciais pelo seu avanço tecnológico e que ainda não existem nos motores gratuitos. Contudo, a decisão em portar para uma plataforma gratuita de desenvolvimento de jogos, licenciar com o fabricante ou disponibilizar como mod será tomada de forma consciente em função da relação custo-benefício. O mais importante é tornar possível a pesquisa educacional otimizando o desenvolvimento e aplicação de novas idéias, reduzindo as exigências de conhecimento de linguagens de programação por parte dos educadores interessados.

\section{Referências Bibliográficas}

ALDRICH, C. Simulations and the future of learning: an innovative (and perhaps revolutionary) approach to e-Learning. San Francisco, CA: Pfeiffer, 2004.

ALDRICH, C. Learning by doing: the essential guide to simulations, computer games, and pedagogy in e-learning and others educational experiences. San Francisco, CA: Pfeiffer, 2005.

AZEVEDO, E. (Coord), Desenvolvimento de jogos 3D e aplicações em realidade virtual. Rio de Janeiro: Elsevier, 2005.

BELL, J.T.; FOGLER H.S. Implementing virtual reality laboratory accidents using the Half-Life game engine, WorldUp, and Java3D. In: PROCEEDINGS OF THE 2003 AMERICAN SOCIETY FOR ENGINEERING EDUCATION ANNUAL CONFERENCE \& EXPOSITION. American Society for Engineering Education, 2003.

BELL, J.T. The VRUPL Lab - Serving education on two fronts. In: SIGCSE ANNUAL CONFERENCE. Norfolk, VA: 2004. 
BORON, D. Infinite regress: the blurring of an architectural game-space. Carleton University. Dissertação de Mestrado. Disponível em: < http://gamasutra.com/ features/20060411/boron_01.shtml>. Acesso em: 1 jun. 2006.

DTI. British Departament of Trade and Industry. Computer games overview. Disponível em: < http://www.dti.gov.uk/sectors/games/index.html>. Acesso em: 4 jun. 2006.

GALVÃO, J.R.; MARTINS P.G.; and GOMES, M.R. Modeling reality with simulation games for a cooperative learning. In: PROCEEDINGS OF THE 2000 WINTER SIMULATON CONFERENCE. San Diego: Society for Modeling and Simulation International, 2000, pp.1692-1698.

GEE, J. P., What video games have to teach us about learning and literacy. New York: Palgrave Macmillan, 2003.

GEE, J. P. Learning by design: Games as learning machines. In: PROCEEDINGS OF GAME DEVELOPERS CONFERENCE. San Jose, CA: 2004.

HECKER, C. Physics in computer games. In: COMMUNICATIONS OF THE ACM. Vol 43, No 7, 2000, pp.34-37.

HOLT, T. Creating a serious mod: development of the GNNVIS virtual forest and Pulse! project visualization mods. In: SERIOUS GAMES SUMMIT DC 2005 SESSIONS. Disponível em: <http://www.cmpevents.com/GDsg05/a.asp?option= $\mathrm{C} \& \mathrm{~V}=11 \&$ SessID =949>. Acesso em 4 ago. 2005.

HOSTETTER, O. Video Games - The necessity of incorporating video games as part of constructivist learning. Game Research. Copenhagen. Dez. 2002. Disponível em: < http://www.game-research.com/art_games_contructivist.asp>. Acesso em 3 jun. 2006.

HOUNSELL, M. S.; GASPARINI, I.; SILVA, R. E.; SUZUKI, R. Integrando textos e ambientes 3D interativos com propósitos educacionais. In: WORKSHOP BRASILEIRO DE JOGOS E ENTRETENIMENTO DIGITAL, 4., 2005, São Paulo. Anais WJOGOS 2005. Porto Alegre: Sociedade Brasileira de Computação, 2005, p.318-323.

ILHA, P. C. A.; CRUZ, D. M. Reality simulation in education: the SimCity in brazilian high school. In: WORKSHOP BRASILEIRO DE JOGOS E ENTRETENIMENTO DIGITAL, 4., 2005, São Paulo. Anais WJOGOS 2005. Porto Alegre: Sociedade Brasileira de Computação, 2005, p.295-299.

LAZZARO, N., Why we play games: Four keys to more emotion in player experiences. Disponível em: <http://www.xeodesign.com/xeodesign_whyweplaygames .pdf> Oakland, CA: XEODesign. Acesso em: 1 ago. 2005.

MAYO, M. J. Ender's game for science and engineering: games for real, for now, or we lose the brain war. In: SERIOUS GAMES SUMMIT DC 2005 SESSIONS. Disponível em: <http://www.seriousgamessummit.com/conference/Speaker\%20 Presentations/ Merrilea_Mayo.ppt>. Acesso em 4 ago. 2005.

MURRAY, J. H. Hamlet on the Holodeck: the future of narrative in Cyberspace. New York: The Free Press, 1997.

NAKAMURA, R. et al. A practical study on the usage of a commercial game engine for the development of educational games. In: PROCEEDINGS OF THE II WORKSHOP DE JOGOS E ENTRETENIMENTO DIGITAL. Salvador: SBC, 2003. (CD-ROM) 
NEVES, F. Mod-games in training and education. In: WORKSHOP BRASILEIRO DE ARTES E DESIGN EM JOGOS DE COMPUTADOR E ENTRETENIMENTO DIGITAL, 2., 2005, São Paulo. Anais SBGAMES 2005 Simpósio Brasileiro de Jogos para Computador e Entretenimento Digital. Porto Alegre: Sociedade Brasileira de Computação. 2005. (CD-ROM).

PERUCIA, A. S.; BERTHÊM, A. C.; BERTSCHINGER, G. L.; MENEZES, R. R. C. Desenvolvimento de jogos eletrônicos - teoria e prática. São Paulo: Novatec, 2005.

SILVA, F. C. L.; SILVA, F. S. C. Um ambiente virtual baseado em jogos para o aprendizado de inteligência artificial. In: WORKSHOP BRASILEIRO DE JOGOS E ENTRETENIMENTO DIGITAL, 4., 2005, São Paulo. Anais WJOGOS 2005. Porto Alegre: Sociedade Brasileira de Computação, 2005, p.284-289.

TAVARES, R.; NEVES, F. Tutorial - Introdução ao Game Design através da modificação de Half-Life. In: WORKSHOP BRASILEIRO DE ARTES E DESIGN EM JOGOS DE COMPUTADOR E ENTRETENIMENTO DIGITAL, 2., 2005, São Paulo. Anais SBGAMES 2005 - Simpósio Brasileiro de Jogos para Computador e Entretenimento Digital. Porto Alegre: Sociedade Brasileira de Computação. 2005. (CDROM).

TORI, R. Game e treinamento profissional. In: SIMPÓSIO GAME_CULTURA. São Paulo: SESC. Disponível em: <http://www.sescsp.org.br/sesc/hotsites/game_cultura /texto_romerotori.doc>. Acesso em: 1 ago. 2005. 